\title{
A unique missense variant in the E1A-binding protein P400 gene is implicated in schizophrenia by whole-exome sequencing and mutant mouse models
}

\author{
Yoshiro Morimoto 1,2, Shinji Ono $\mathbb{1}^{1,3}$, Shintaro Yoshida ${ }^{1,2}$, Hiroyuki Mishima $\mathbb{1}^{3}$, Akira Kinoshita ${ }^{3}$, Takeshi Tanaka ${ }^{4}$, \\ Yoshihiro Komohara ${ }^{5}$, Naohiro Kurotaki ${ }^{6}$, Tatsuya Kishino ${ }^{7}$, Yuji Okazaki ${ }^{8}$, Hiroki Ozawa ${ }^{1,2}$, Koh-ichiro Yoshiura $\mathbb{1}^{3}$ and \\ Akira Imamura ${ }^{1,2}$
}

\begin{abstract}
Genetic and epidemiological evidence has suggested that genetic factors are important in schizophrenia, although its pathophysiology is poorly understood. This study used whole-exome sequencing to investigate potential novel schizophrenia-causing genes in a Japanese family containing several members affected by severe or treatmentresistant schizophrenia. A missense variant, chr12:132064747C C T (rs200626129, P2805L), in the E1A-binding protein P400 (EP400) gene completely segregated with schizophrenia in this family. Furthermore, numerous other EP400 mutations were identified in the targeted sequencing of a schizophrenia patient cohort. We also created two lines of Ep400 gene-edited mice, which had anxiety-like behaviours and reduced axon diameters. Our findings suggest that rs200626129 in EP400 is likely to cause schizophrenia in this Japanese family, and may lead to a better understanding and treatment of schizophrenia.
\end{abstract}

\section{Introduction}

Schizophrenia is a severe and chronic neuropsychiatric disorder ${ }^{1,2}$ with numerous psychotic symptoms, including hallucinations and delusions ${ }^{3-6}$. Schizophrenia is usually treated with antipsychotics, although $\sim 30 \%$ of cases respond poorly, which is termed treatment-resistant schizophrenia $(\mathrm{TRS})^{1,7}$. Because of these clinical aspects, schizophrenia is a major cause of global disease burden ${ }^{8}$.

The pathophysiology of schizophrenia remains unclear. A number of genetic and epidemiological studies suggest that genetic factors play an important role in its pathogenesis, and its heritability estimate is $0.81^{9}$. Although genome-wide

Correspondence: Shinji Ono (shinji1231zzz@yahoo.co.jp) or Kohichiro Yoshiura (kyoshi@nagasaki-u.ac.jp)

'Department of Neuropsychiatry, Unit of Translation Medicine, Nagasaki University Graduate School of Biomedical Sciences, Nagasaki, Japan

${ }^{2}$ Child and Adolescent Psychiatry Community Partnership Unit, Nagasaki University Hospital, Nagasaki, Japan

Full list of author information is available at the end of the article association studies (GWAS) have identified >150 schizophrenia-associated polymorphisms ${ }^{10}$, no specific variants with direct functional effects have been reported ${ }^{11}$. Furthermore, no common variant has an individually large effect, which is expected considering the selection pressures of impaired reproductive fitness associated with schizophrenia, and considering that the majority of risk cannot be accounted for by the associated genes reported to date ${ }^{12,13}$. Rare genetic variants detected by whole-exome sequencing (WES) or whole-genome sequencing (WGS) using nextgeneration sequencing (NGS) have demonstrated that the RNA-binding motif 12 (RBM12) gene, the SET domain containing $1 \mathrm{~A}$ (SETD1A) gene, and the solute carrier family 6 member 1 (SLC6A1) gene are high-risk factors ${ }^{14-17}$. The functional consequences of these rare variants are expected to be more readily interpretable ${ }^{18}$.

Using WES, the present study identified a unique missense variant in the E1A-binding protein P400 (EP400)

\section{(c) The Author(s) 2021}

(c) (i) Open Access This article is licensed under a Creative Commons Attribution 4.0 International License, which permits use, sharing, adaptation, distribution and reproduction cc) in any medium or format, as long as you give appropriate credit to the original author(s) and the source, provide a link to the Creative Commons license, and indicate if changes were made. The images or other third party material in this article are included in the article's Creative Commons license, unless indicated otherwise in a credit line to the material. If material is not included in the article's Creative Commons license and your intended use is not permitted by statutory regulation or exceeds the permitted use, you will need to obtain permission directly from the copyright holder. To view a copy of this license, visit http://creativecommons.org/licenses/by/4.0/. 
gene in a Japanese family in which several members were affected by severe schizophrenia/TRS. Next, the frequencies of rare EP400 variants were compared between sporadic schizophrenia cases and controls. We also generated Ep400 gene-edited mice using clustered regularly interspaced short palindromic repeats (CRISPR)/Cas9. These mice developed anxiety-like behaviours and ultrastructural abnormalities in the central nervous system (CNS). We report here an EP400/Ep400 variant that elicited abnormalities in neurobehaviour, which may lead to schizophrenia.

\section{Subjects and methods}

\section{Clinical presentation in a Japanese family}

Three first-generation family members were diagnosed with schizophrenia. One (Fig. 1a, I-9) had consulted a psychiatric hospital because of auditory hallucinations and delusions at 47 years old and was diagnosed with schizophrenia. Her elder sister and brother (I-3 and I-4, respectively) also had auditory hallucinations and delusions and were hospitalised more than once; however, their medical records were not preserved. Seven secondgeneration family members (II-2, II-3, II-5, II-10, II-12, II13, and II-17) were diagnosed with schizophrenia and treated with antipsychotic medications. Two of these second-generation patients (II-3 and II-17) were diagnosed with TRS $^{19}$ and required multiple long-term hospitalisations, but were not treated with clozapine or electroconvulsive therapy. Furthermore, two thirdgeneration family members were diagnosed with schizophrenia (III-2 and III-3). III-2 refused medical care and medication. None of the patients had neurological abnormalities by standard neurological exams or comorbidities. All subjects in the family were evaluated by two or more psychiatrists. Diagnoses of psychiatric diseases were made with reference to the International Classification of Diseases, Tenth Revision (ICD-10) and the Diagnostic and Statistical Manual of Mental Disorders, Fifth Edition (DSM-5). A detailed clinical presentation of the Japanese family is summarised in Table 1.

\section{DNA extraction}

Of the 10 family members recruited, 7 were affected (I9, II-3, II-5, II-12, II-17, II-2, and III-3) and 3 were unaffected (I-13, II-11, and II-20) by schizophrenia (Fig. 1a). After obtaining written consent, $10 \mathrm{~mL}$ of peripheral blood was collected from individuals. Genomic DNA was extracted from peripheral blood leukocytes using a QIAamp DNA Midi Kit (Qiagen, Hilden, Germany).

\section{WES}

We assessed DNA quality using a NanoDrop 2000 (Thermo Fisher Scientific, Waltham, MA, USA) and a Qubit fluorometer for nucleic acid quantification
(Thermo Fisher Scientific). We performed WES for the seven affected and three unaffected individuals (Fig. 1a) using a SureSelect Exome Target Enrichment System v5 (Agilent Technologies, Santa Clara, CA, USA) followed by paired-end sequencing on a HiSeq 2500 (Illumina, San Diego, CA, USA). Mean depths and coverage rates are summarised in Supplementary Table 1.

\section{Sequencing data analysis}

Fastq format files were generated using bcl2fastq software. Novoalign software (Novocraft Technologies, Kuala Lumpur, Malaysia) was used to align reads on the hg38/GRCh38 human reference genome sequence. Aligned reads were sorted using Novosort software (Novocraft Technologies) and subjected to polymerase chain reaction (PCR) marking and optical duplication using MarkDuplicates in the Picard tools package (http://broadinstitute.github.io/picard/). The Genome Analysis Toolkit (GATK) ${ }^{20}$ was used to perform local realignment (GATK IndelRealigner), and variant calling (GATK HaplotypeCaller) was implemented in an inhouse workflow management tool ${ }^{21}$. Single nucleotide variations (SNVs) and insertions/deletions (indels) were annotated using ANNOVAR software ${ }^{22}$.

\section{WES variant filtering}

Substitutions meeting the following criteria were considered 'deleterious': (1) mutations: stop-gain, stop-loss, nonsynonymous, or splice-site mutations according to GENCODE basic 33, downloaded from the UCSC Genome Browser (https://genome.ucsc.edu); and (2) alternative allele frequencies of $\leq 0.5 \%$ at mutation loci in the following databases: 4.7KJPN data of allele and genotype frequency panels from 4773 Japanese individuals (https://jmorp. megabank.tohoku.ac.jp/202001/variants); genome aggregation database (gnomAD) v2.1.1 and v3 (https://gnomad. broadinstitute.org); ${ }^{23}$ and Human Genomic Variation Database (HGVD) v2.3 exome data from Japanese individuals (http://www.hgvd.genome.med.kyoto-u.ac.jp/index. $h t m l)^{24}$. Two deleterious variants were shared among the affected individuals (Supplementary Table 2). We excluded rs768213554 in strawberry notch homolog 1 (SBNO1) gene from the candidates because it was found in the cohort of 445 Japanese controls. Consequently, we considered the rs200626129 variant in EP400 as the potential pathogenic variant of our family. The WES variant filtering process is summarised in Supplementary Table 3.

\section{Sanger sequencing}

Sanger sequencing confirmed that one variant was shared among all of the affected individuals and was absent in the three unaffected individuals. Primers were designed using Primer $3:^{25}$ chr12:132064747; C $>\mathrm{T}$, forward, 5'-TCATCAAAATGCAGAAGCAGA-3', reverse, 5'-TCATCAAAATGCAGAAGCAGA-3'. Genomic DNA 
a

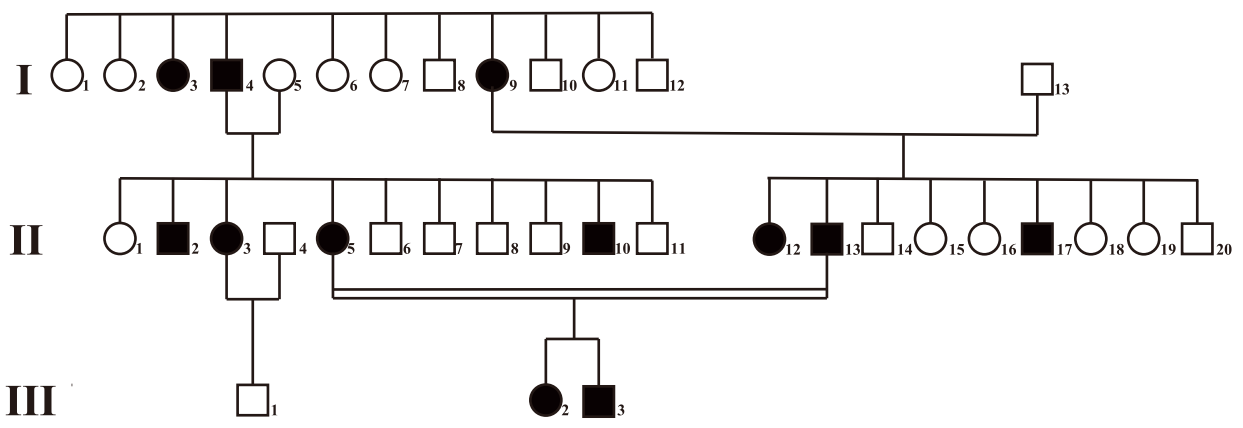

b

EP400: p.Pro2805Leu

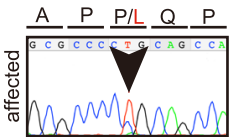

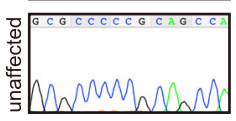

C

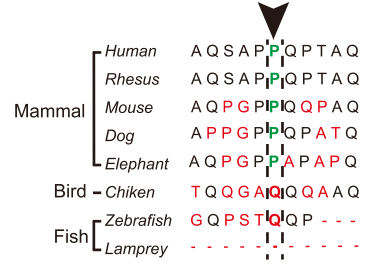

d

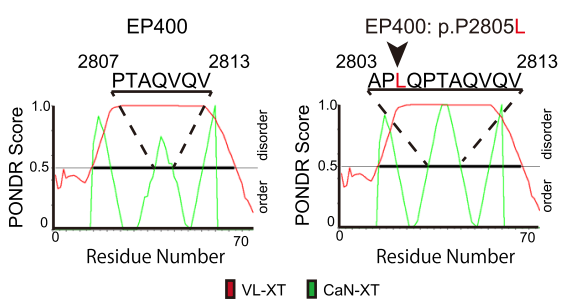

e

\section{EP400: ENST00000389561.6}

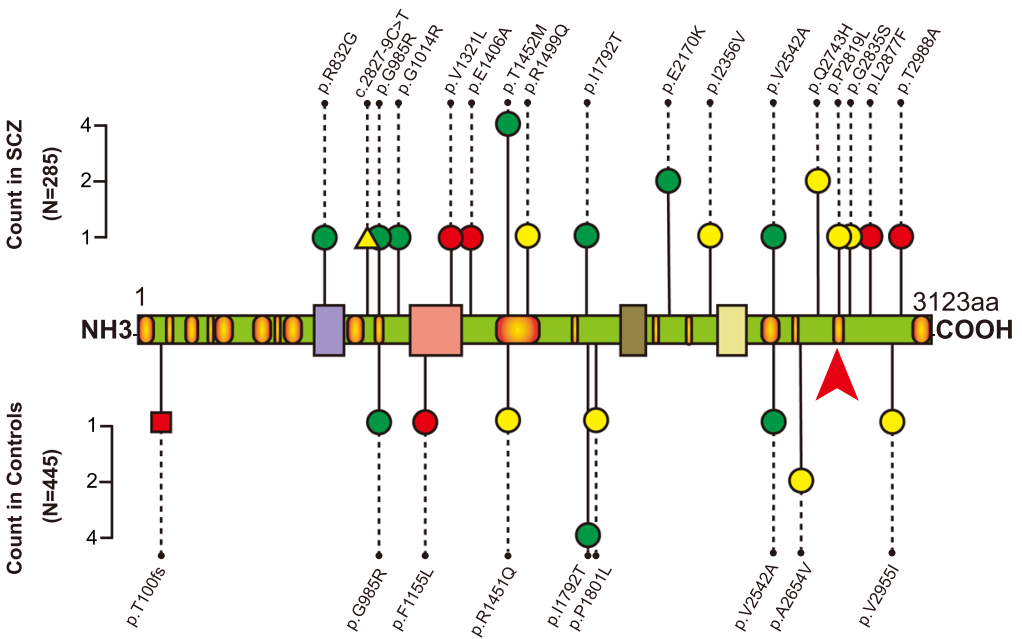

\section{Disordered Binding Regions $\square$ Helicase/SANT-associated (HSA) domain $\square$ SNF2-related,N-terminal domain \\ $\square$ Myb-like domain $\square$ Helicase C-terminal domain \\ Novel missense variant $\square$ Novel loss of function variant \\ Familial mutation: p.P2805L} OUltra-rare missense variant $\Delta$ Ultra-rare spling variant

Rare missense variant

Fig. 1 Japanese family with multiple individuals affected by schizophrenia. a Japanese family with multiple individuals affected by schizophrenia. Black symbols represent individuals diagnosed with schizophrenia. b Segregation of the missense mutation (chr12: 132064747; C>T on hg38, p.P2805L in ENST00000389561.7) in EP400 with schizophrenia. c The position of the familial missense mutation in the EP400 gene. $\mathbf{d}$ The CaN-XT algorithm in PONDR was used to predict the effect of p.P2805L on the EP400 protein. e EP400 deleterious variants in Japanese individuals with or without schizophrenia. Rare variants are represented by lollipops, and counts of alleles with variants in the cases (top panel) and controls (bottom panel) are shown. Novel variant = novel variant in all databases; ultra-rare variant = variants with MAF $<0.001$ in any database; rare variant = variants with $0.001<$ MAF $<0.005$ in any database. 
Table 1 Clinical presentation of the Japanese family including multiple patients.

\begin{tabular}{|c|c|c|c|c|c|c|c|c|c|c|c|}
\hline \multirow[t]{2}{*}{ ID } & \multirow[t]{2}{*}{ Age of onset (years) } & \multicolumn{2}{|c|}{ Diagnosis } & \multicolumn{5}{|c|}{$\begin{array}{l}\text { Clinician-rated } \\
\text { dimensions of } \\
\text { psychosis symptom } \\
\text { severity }\end{array}$} & \multirow[t]{2}{*}{ GAF } & \multirow[t]{2}{*}{ Medications } & \multirow[t]{2}{*}{ Other } \\
\hline & & ICD-10 & DSM-5 & I & II & III & IV & $\mathbf{v}$ & & & \\
\hline $1-9$ & 47 & F20.00 & 295.90 & 2 & 2 & 3 & 1 & 3 & 45 & $H P D^{a}$ & - \\
\hline$\|-3$ & 21 & F20.00 & 295.90 & 3 & 3 & 4 & 1 & 4 & 15 & HPD, ZTN, CHP, OLZ, QUE, RIS & TRS \\
\hline$\|-5$ & 34 & F20.04 & 295.90 & 3 & 2 & 2 & 1 & 3 & 45 & RIS*, OLZ, QUE & - \\
\hline$\|-12$ & 18 & F20.00 & 295.90 & 2 & 2 & 2 & 0 & 3 & 45 & HPD*, OLZ, QUE, RIS & - \\
\hline$\|-17$ & 17 & F20.10 & 295.90 & 3 & 3 & 4 & 2 & 4 & 15 & HPD, OLZ, QUE, RIS & TRS \\
\hline|| $\mid-2$ & 17 & F20.10 & 295.90 & 1 & 1 & 3 & 1 & 4 & 35 & - & No consent for drug treatment \\
\hline|| $\mid-3$ & 30 & F20.01 & 295.90 & 2 & 2 & 1 & 0 & 2 & 65 & OLZ ${ }^{\mathrm{a}}$ & - \\
\hline
\end{tabular}

Diagnosis: ICD-10: International Statistical Classification of Diseases and Related Health Problems, 10 ${ }^{\text {th }}$ edition, DSM-5: Diagnostic and Statistical Manual of Mental Disorders, 5th Edition; F20.00: paranoid schizophrenia, continuous; F20.01: paranoid schizophrenia, episodic with progressive defect; F20.04: paranoid schizophrenia, incomplete remission; F20.10: hebephrenic schizophrenia, continuous; 295.90: schizophrenia. Clinician-Rated Dimensions of Psychosis Symptom Severity: I: hallucinations, II: delusions, III: disorganised speech, IV: abnormal psychomotor behaviour, V: negative symptoms. GAF: Global Assessment of Functioning Scale. Medications: HPD: haloperidol, ZTN: zotepine, CHP: chlorpromazine, OLZ: olanzapine, QUE: quetiapine, RIS: risperidone. Other: TRS: treatment-resistant schizophrenia. ${ }^{\mathrm{a}}$ Drugs that improved the GAF scale over 40.

(5 ng) was amplified using KOD FX Neo polymerase (Toyobo, Osaka, Japan) by PCR in $20-\mu \mathrm{L}$ reactions. PCR products were purified using AMPureXP® (Agencourt, Beverly, MA, USA) and sequencing reactions were performed using a BigDye Terminator Cycle Sequencing Kit v3.1 (Applied Biosystems, Foster City, CA, USA). After purification using CleanSEQ (Agencourt), DNA fragments were run and separated on an ABI Genetic Analyzer 3130 (Applied Biosystems).

\section{Mutational analysis of Japanese inpatient schizophrenia case-control groups}

Samples from 285 sporadic, inpatient schizophrenia patients in Japan, who had been diagnosed by psychiatrists in accordance with the DSM-5 or ICD-10, and 445 control samples were collected. For the schizophrenia association test using variants in the EP400 locus, we performed target sequencing using a SeqCap EZ Choice Library (Roche NimbleGen, Madison, WI, USA) according to the manufacturer's protocol. Prepared libraries were sequenced using a HiSeq2500 (Illumina). Subsequent analyses were performed using the hg19/GRCh37 human reference genome sequence, following the "sequencing data analysis" section. CrossMap (http:// crossmap.sourceforge.net) ${ }^{26}$ was used to convert data to hg38/GRCh38 human reference genome sequence assemblies.

\section{In silico analysis of rs200626129 in EP400}

To analyse potential functional changes in the EP400 protein, mutation significances were scored using SIFT $^{27}$,
PROVEN $^{28}$, MutationTaster ${ }^{29}$, and M-CAP ${ }^{30}$. The Predictor of Natural Disordered Regions (PONDR) algorithm was used to predict disordered protein regions (http:// www.pondr.com/pondr-tut1.html $)^{31-33}$.

\section{Single-locus association test with deleterious variants}

To identify unique deleterious variants in EP400 that were associated with schizophrenia, we tested the accumulation of 23 deleterious variants identified by target sequencing. We used Fisher's exact test to estimate $P$-values. The significance threshold was 0.002174, with Bonferroni correction.

\section{Gene-based rare-variant association tests}

To test differences in the burden of the candidate gene, we used Efficient and Parallelizable Association Container Toolbox (EPACTS) algorithms, which provide three different analyses (combined multivariate and collapsing $[\mathrm{CMC}]^{34}$, Madsen-Browning ${ }^{35}$, and optimal sequence kernel association test [SKAT-O $]^{36}$ ). We excluded common variants with alternative allele frequencies $>0.005$ in any public database (1000 Genomes Project, all population data released October 2014; ${ }^{37}$ NIH NHLBI 6515 exome data [http://evs.gs.washington.edu/EVS/]; Exome Aggregation Consortium 65000 exome data; ${ }^{38}$ HGVD exome data of 1208 individuals in Japan; ${ }^{39}$ and SNV allele frequency in whole-genome sequencing data of 2049 healthy Japanese individuals [https://ijgvd.megabank. tohoku.ac.jp]) and $>0.005$ in our in-house control samples, with a call rate $<90 \%$ or genotyping quality $<99$. We selected four histone-modifying genes (the histone 
deacetylase $4[H D A C 4]$ gene, the lysine methyltransferase $2 \mathrm{D}[K M T 2 D]$ gene, the lysine demethyltransferase $6 \mathrm{~A}$ $[K D M 6 A]$ gene, and the nuclear receptor binding SET domain protein 1 [NSD1] gene) as controls. The significance threshold was set to 0.01, with Bonferroni correction.

\section{Generation of Ep400 knock-in mice}

We designed guide RNA onto exon 47 of mouse ortholog Ep400, corresponding to the mutation position in the family from the present study. Single-guide RNA was designed using the ATUM gRNA Design Tool (https://www.atum. bio/eCommerce/cas9/input) as follows: 5'-CACTTGC GCTGGTTGCTGTG-3' ${ }^{\prime}$. Single-strand DNA oligonucleotides (ssODN) to introduce mutations into mouse DNA were as follows: \#1: 5'-CCACCACAGCCCCCACCACC GCAGGCGCAGCCAGGTCCCCTACAGCAACCAGCGC AAGTGCAAGTACAGACTCCACAGCC-3' , \#2: 5'-GATG CAGCTGCCACCACAGCCCCCACCACCGCAGGCCCA GCCAGGTCCTCTACAGCAACCAGCGCAAGTGCAAG TACAGACTCCACAGCCCCCACAGCAA-3'.

Single-guide RNA, crRNA, tracrRNA, HiFi Cas9 (IDT Inc, Coralville, IA, USA), and ssODN (FASMAC, Kanagawa, Japan) were mixed according to the mouse zygote microinjection protocol provided by IDT Inc., and were then injected into $\mathrm{C} 57 \mathrm{BL} / 6 \mathrm{~N}$ zygotes using a microinjector (Olympus IX70 Fluorescence Microscope Cutaway, Olympus, Tokyo, Japan), TransferMan NK2 (Eppendorf, Hamburg, Germany), FemtoJet 4i (Eppendorf), and CellTram Oil (Eppendorf). Next, zygotes were cultured until the two-cell stage in vitro and transferred to recipient pseudopregnant female oviducts. PCR was used to verify genotypes from mouse DNA using KOD FX Neo polymerase (Toyobo). The PCR primers were as follows: forward, 5'-TGGAGGGTGGCTTATGGTTA-3'; reverse, 5'-GTCACTGTGGTGCCTGTGAG-3'.

\section{Histological analysis}

Fresh mouse brains and spinal cords were fixed in 10\% formalin for $72 \mathrm{~h}$, embedded in paraffin, and sectioned at $5-\mu \mathrm{m}$ intervals. After deparaffinisation, slides were stained with haematoxylin and eosin (HE) to visualise structures. The Klüver-Barrera (KB) stain was used to detect myelin sheaths. We viewed and photographed sections using a BZ-9000 All-in-One Fluorescence Microscope (Keyence, Osaka, Japan). For immunohistochemical staining, tissue slides were deparaffinised, rehydrated, and rinsed in Trisbuffered saline (TBS). For antigen retrieval, tissue sections were microwaved for $20 \mathrm{~min}$ in $10 \mathrm{mM}$ citrate buffer using an MI-77 microwave processor (Azumaya Corporation, Tokyo, Japan). Sections were then blocked in Protein Block Serum-Free (Dako, Carpinteria, CA, USA) for $1 \mathrm{~h}$ at room temperature, followed by incubation with primary and secondary antibodies for $1 \mathrm{~h}$ each at room temperature. Immunofluorescent staining was performed using the following primary antibodies: polyclonal antiEP400 (HPA016704, Atlas Antibodies, Bromma, Sweden) and polyclonal anti-MAP2 (ab5392, Abcam, Cambridge, UK). Secondary antibodies were goat anti-chicken IgY H\&L Alexa Fluor 488 (ab150169, Abcam) and goat antirabbit IgG H\&L Alexa Fluor 555 (ab150078, Abcam). Primary and secondary antibodies were diluted using Antibody Diluent with Background Reducing Components (Dako). Sections were mounted with Vectashield Mounting Medium for fluorescence analysis with DAPI (Vector Laboratories, Burlingame, CA, USA). Fluorescent images were taken using a BZ-9000 All-in-One Fluorescence Microscope (Keyence).

\section{Transmission electron microscopy (TEM)}

Samples were fixed with $2 \%$ glutaraldehyde (Nacalai Tesque, Kyoto, Japan) in $0.1 \mathrm{M}$ sodium cacodylate buffer containing $1 \mathrm{mM} \mathrm{CaCl} 2$ and $1 \mathrm{mM} \mathrm{MgCl}_{2}$ (cacodylate buffer, $\mathrm{pH}$ 7.4) at room temperature for $72 \mathrm{~h}$, rinsed with cacodylate buffer, and post-fixed with $1 \% \mathrm{OsO}_{4}$ (Nacalai Tesque) in cacodylate buffer at $4{ }^{\circ} \mathrm{C}$ for $60 \mathrm{~min}$. Next, samples were washed with cacodylate buffer, dehydrated in a graded series of ethanol and acetone, and embedded in Quetol 651 epoxy resin (Nisshin EM, Tokyo, Japan). The blocks were then sectioned at $5-\mu \mathrm{m}$ intervals and stained with toluidine blue (TB) to visualise myelin sheaths. Resin-embedded samples were further trimmed and sectioned using a diamond knife on an ultramicrotome (Reichert-Jung, Vienna, Austria). Ultra-thin sections were collected on grids, stained with uranyl acetate and lead citrate, and examined at $80 \mathrm{kV}$ by TEM (JEM-1230; JEOL, Tokyo, Japan). Axon diameters and Gratios of randomly selected axons in the white matter of the spinal cord were measured using ImageJ (axon diameter, $n=100$; G-ratio, $n=30)^{40,41}$. The two-sided $F$-test was applied to confirm normally distributed data. The two-sided Student's $t$-test and two-sided Welch's $t$-test were applied for comparison. The significance threshold was set to 0.05 .

\section{Neurobehavioural screening battery in Ep400 knock-in mice}

The mice used in the present study were derived from backcrossing to C57BL/6N for five generations. Six male Ep400-p.P2715L\#1 mice and six male wild-type (WT) littermates were examined using behavioural tests at 3 and 6 months old. Mice were housed individually at $22{ }^{\circ} \mathrm{C}-26{ }^{\circ} \mathrm{C}$, humidity $40 \%-65 \%$, and 12 -h light/dark periods. Hindlimb clasping: a marker of disease progression in a number of mouse models of neurodegeneration $^{42,43}$. We evaluated hindlimb clasping scores using the following criteria ${ }^{44}$. Hindlimbs were consistently splayed outward, away from the abdomen $=0$. One hindlimb was 
retracted toward the abdomen for more than $50 \%$ of the time $\operatorname{suspended}=1$. Both hindlimbs were partially retracted toward the abdomen for more than $50 \%$ of the time suspended $=2$. Hindlimbs were entirely retracted and touching the abdomen for more than $50 \%$ of the time suspended $=3$. Open-field test: measures locomotor activity, exploratory drive, neophobia, and certain aspects of anxiety ${ }^{45}$. Mice were placed in a clean box measuring $50 \times 50 \times 40 \mathrm{~cm}$. The central area was $60 \%$ of the area. After $15 \mathrm{~min}$ resting time, mice were placed peripherally in the box facing outward, and were observed for $10 \mathrm{~min}$ by video tracking. Video data were analysed using BaraBaby X (LNSOFT, Tokyo, Japan). Migration length $(\mathrm{cm})$ was measured as horizontal activity. Centre time was calculated as the ratio of movement time in the central area to the entire movement time. Social interaction test: evaluates social behaviour phenotypes by placing two animals in a neutral cage and measuring the percentage of time spent in direct physical contact, including anogenital exploration, sniffing with direct contact, crawling, grooming, and play behaviour ${ }^{46}$. Mice were kept in cages for 7 days to prepare, and stimulator mice were then put into the same cage as the subject mice under video monitoring. After $15 \mathrm{~min}$, stimulator mice were removed, and video monitoring was stopped. Movies were analysed using BORIS Software 4.1.4 (https://www.boris.unito.it) ${ }^{47}$. $Y$-maze test: evaluates short-term spatial working memory $^{48}$. After 15 min resting time, mice were placed on one corner of an arm of the apparatus, facing the outer side, under video monitoring. Mice were monitored for $10 \mathrm{~min}$ without stimulation, and the number of entries into each of three arms was counted. Spontaneous alternations were calculated if the mouse entered three different arms sequentially. Rotarod test: evaluates motor coordination ${ }^{49}$. Mice in a "stable state" after 20 min rest were placed on the rotarod (Rota-Rod Treadmill for Mice, Model MK610A, Muromachi Kikai, Tokyo, Japan) at $4 \mathrm{rpm}$. After several seconds, the rotor accelerated to $40 \mathrm{rpm}$ for $300 \mathrm{~s}$. Tests were repeated up to three times if mice fell off before $10 \mathrm{~s}$ had passed (Trial 1). After $20 \mathrm{~min}$, the same tests were again administered (Trial 2). An two-sided $F$ test confirmed normally distributed data and two-sided Student's $t$-test was used for two-sample significance tests. The significance threshold was set to 0.05. All behavioural testing was performed by Unitech Co., Ltd. (Chiba, Japan).

\section{Results}

\section{WES and variant filtering}

We identified a large Japanese family with autosomal dominant inheritance of schizophrenia. The patients in this family exhibited severe negative symptoms and low scores in the Global Assessment of Functioning Scale (Clinician-Rated Dimensions of Psychosis Symptom Severity: $V_{\text {average }}=3.29$; Global Assessment of Functioning Scale: $G_{A F} F_{\text {average }}=37.9$ ). Of the patients in this family, two second-generation TRS patients (II-3 and II17) responded poorly to antipsychotics and required multiple long-term hospitalisations (Table 1). To identify potential pathogenic genetic variants, we performed WES on 10 family members (affected: I-9, II-3, II-5, II-12, II-17, III-2, III-3; unaffected: I-13, II-11, II-20; Fig. 1a). Using NGS, we detected 148,069 variants, of which 2176 variants were annotated to be deleterious (Supplementary Table 3). Of the 2176 deleterious variants, only two variants, on chromosome $12 \mathrm{q} 24.3$, were completely segregated with schizophrenia (chr12:123311084, T>C [rs768213554] in SBNO1; and chr12:132064747, C>T [rs200626129] in EP400; Supplementary Tables 2, 3). Next, we screened these two substitutions in 445 healthy Japanese individuals and found that rs768213554 in SBNO1 gene was present in healthy controls, as well as in two Japanese public databases (control cohort: minor allele frequency $[\mathrm{MAF}]=0.0022$; HGVD v2.30: $\mathrm{MAF}=$ $0.00124 ; 4.7 \mathrm{KJPN}$ : MAF $=0.0012$ ). Because the pathogenic mutation in this family was expected to have an extremely high effect size and penetrance rate of Mendelian inheritance, we excluded rs768213554 in SBNO1 from the candidate variants. Notably, mutation screening indicated that rs200626129 in EP400 (confirmed by direct sequencing; Fig. 1b) was a 'unique' variant, because it was a novel variant in both healthy controls and Japanese databases (control cohort: MAF $=$ none; HGVD2.30: $\mathrm{MAF}=$ none; $4.7 \mathrm{KJPN}: \quad \mathrm{MAF}=$ none; Supplementary Tables 2, 3).

The position of the familial mutation, in exon 48 of the EP400 gene, was located within a disordered binding region that is highly conserved among mammals (Fig. 1c). We next analysed whether rs200626129 in EP400 affects protein function, using SIFT, PROVEN, MutationTaster, and M-CAP. All programs evaluated the variant as 'damaging' (SIFT score $=0.007$, deleterious; PROVEN score $=-2.67$, deleterious; MutationTaster score $=0.994$, disease-causing; $\mathrm{M}$-CAP score $=0.254$, damaging). The CaN-XT algorithm in PONDR predicted that the variant would elongate the disordered region of the EP400 protein (Fig. 1d). These findings suggest that rs200626129 in EP400 causes dysfunction of the protein, and that this variant is relevant to the pathogenesis of schizophrenia.

The rs200626129 variant in EP400 was considered to be the pathogenic variant in this family because of its uniqueness and complete linkage, although its genetic burden in the development of schizophrenia and its pathogenic effects on CNS functions were not evaluated. 
Table 2 Rare variants in gene-based burden tests for EP400.

\begin{tabular}{|c|c|c|c|c|c|c|c|}
\hline \multirow[t]{2}{*}{ Gene } & \multicolumn{2}{|c|}{$\begin{array}{l}\text { Count of rare deleterious } \\
\text { variants }\end{array}$} & \multirow[t]{2}{*}{$\begin{array}{l}\text { Frequency of rare variants in } \\
\text { SCZ subjects }(n=285)\end{array}$} & \multirow[t]{2}{*}{$\begin{array}{l}\text { Frequency of rare variants in } \\
\text { control subjects }(n=445)\end{array}$} & \multicolumn{3}{|c|}{$P$-value } \\
\hline & $\begin{array}{l}S C Z \\
(n=285)\end{array}$ & $\begin{array}{l}\text { Controls } \\
(n=445)\end{array}$ & & & $\mathrm{CMC}$ & Madsen-Browning & SKAT-O \\
\hline EP400 & 21 & 14 & $7.37 \%$ & $3.15 \%$ & 0.059 & 0.051 & 0.011 \\
\hline HDAC4 & 6 & 8 & $2.11 \%$ & $1.80 \%$ & 0.955 & 0.959 & 0.36 \\
\hline KMT2D & 35 & 37 & $12.28 \%$ & $8.31 \%$ & 0.358 & 0.288 & 0.194 \\
\hline KDM6A & 9 & 7 & $3.16 \%$ & $1.57 \%$ & 0.304 & 0.295 & 0.395 \\
\hline NSD1 & 13 & 18 & $4.56 \%$ & $4.04 \%$ & 0.644 & 0.648 & 0.834 \\
\hline
\end{tabular}

The significance threshold was 0.01 , with Bonferroni correction. SCZ: schizophrenia; CMC: combined multivariate and collapsing; SKAT-O: optimal sequence kernel association test.

Single-locus association test with the deleterious variants identified by EP400 sequencing

To evaluate the MAF of rs200626129 in sporadic schizophrenia, and to identify other unique deleterious variants in EP400 that are associated with schizophrenia, we performed target capture sequencing of EP400 in Japanese individuals with or without schizophrenia (schizophrenia: $n$ $=285$; controls: $n=445$ ). Notably, rs200626129 in EP400 was not identified in the 285 schizophrenia patients.

Nevertheless, we identified 23 deleterious variants in EP400 using targeted sequencing. Of these 23 variants, 6 were novel variants that were not registered in databases (HGVD2.30, 4.7KJPN, or gnomAD exome), 10 were ultrarare variants that were registered in at least one database with $\mathrm{MAF}<0.001$, and 7 were rare variants registered in at least one database with $0.001<\mathrm{MAF}<0.005$. None of the 23 single loci had a significant association with schizophrenia (Fig. 1e, Supplementary Table 4).

\section{Gene-based rare-variant association tests}

Schizophrenia patients had a tendency toward a higher frequency of deleterious variants $(7.37 \%)$ compared with controls (3.15\%; Table 2). To confirm the higher frequency of deleterious variants in EP4OO in patients with schizophrenia compared with controls, we performed gene-based rare-variant association tests using EPACTS algorithms. We selected four histone-modifying genes (HDAC4, KMT2D, KDM6A, and NSD1) as controls. In the gene-based rare-variant association tests, EP400 showed the lowest $P$-values in the five tested genes, but did not show significant enrichment of rare variants in schizophrenia (CMC, $P=0.059184$; Madsen-Browning, $P=$ 0.051225; SKAT-O, $P=0.010889$; Table 2).

\section{Generation of Ep400-p.P2715L mice using CRISPR/Cas9 technology}

To reveal the functional significance of the familial mutation in brain morphology and behavioural characteristics, we generated two lines of Ep400 ${ }^{\mathrm{P} 2715 \mathrm{~L} /}$ P2715L mice (Ep400-p.P2715L\#1 and \#2), corresponding to the human P2805L mutation, using CRISPR/Cas9. The Ep400-p.P2715L\#1 mice were born at a predicted ratio by Mendelian segregation (Supplementary Table 5) and did not show any differences in body size compared with controls (3 months old: Student's $t$-test, $P=0.1304$; 6 months old: Student's $t$-test, $P=0.07203$; Fig. 2a, Supplementary Table 6). According to public databases, the EP400 protein is expressed in the brains of both mice and humans ${ }^{50,51}$. Furthermore, our immunofluorescent staining results confirmed the expression of EP400 in 3month-old mouse brains, especially in neural cell nuclei. However, there were no differences in EP400 protein expression patterns between the knock-in mice and their WT littermates (Supplementary Fig. 1).

\section{Screening analysis for the known phenotypes of Ep400} conditional-knockout mice (hindlimb clasping and myelin abnormalities) using Ep400-p.P2715L mice

Recent studies have reported distinctive phenotypes in Ep400 conditional-knockout mice (notably, hindlimb clasping and myelin abnormalities) ${ }^{52,53}$. Thus, to investigate the possibility that the p.P2715L mutation also causes Ep400 protein dysfunction in vivo, we screened our Ep400-p.P2715L mice for these known phenotypes of Ep400 conditional-knockout mice.

The Ep400-p.P2715L\#1 mice showed peculiar hindlimb clasping from 3 months of age (hindlimb clasping score, $\mathrm{WT}_{\text {average }}=0.5 ; \quad$ Ep400-p.P2715L\# $1_{\text {average }}=1.833 ;$ Student's $t$-test, $P=0.046$; Fig. $2 \mathrm{~b}$, Supplementary Table 7, Supplementary Video 1), which is a sign that is observed in mice with CNS dysfunction and is used as a marker of disease progression in a number of mouse models of neurodegeneration ${ }^{42,43}$. This behaviour was also present in Ep400-p.P2715L\#2 mice (hindlimb clasping score, Ep400-p.P2715L\# $2_{\text {average }}=2$; Student's $t$-test, $P=0.023$; Supplementary Fig. 2a, Supplementary Table 7). 
a

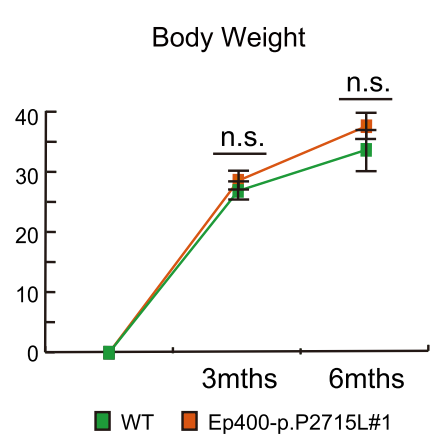

C

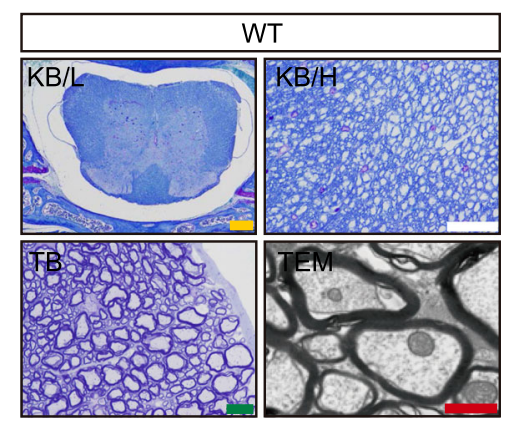

d

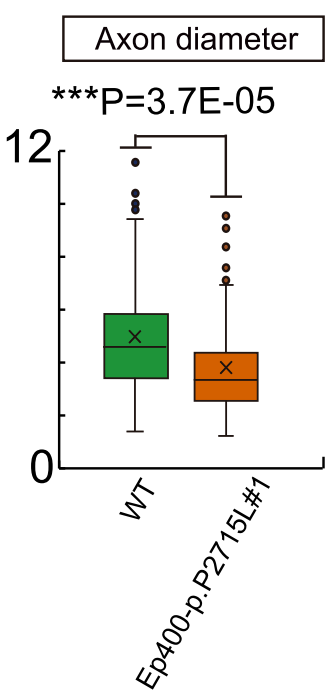

b
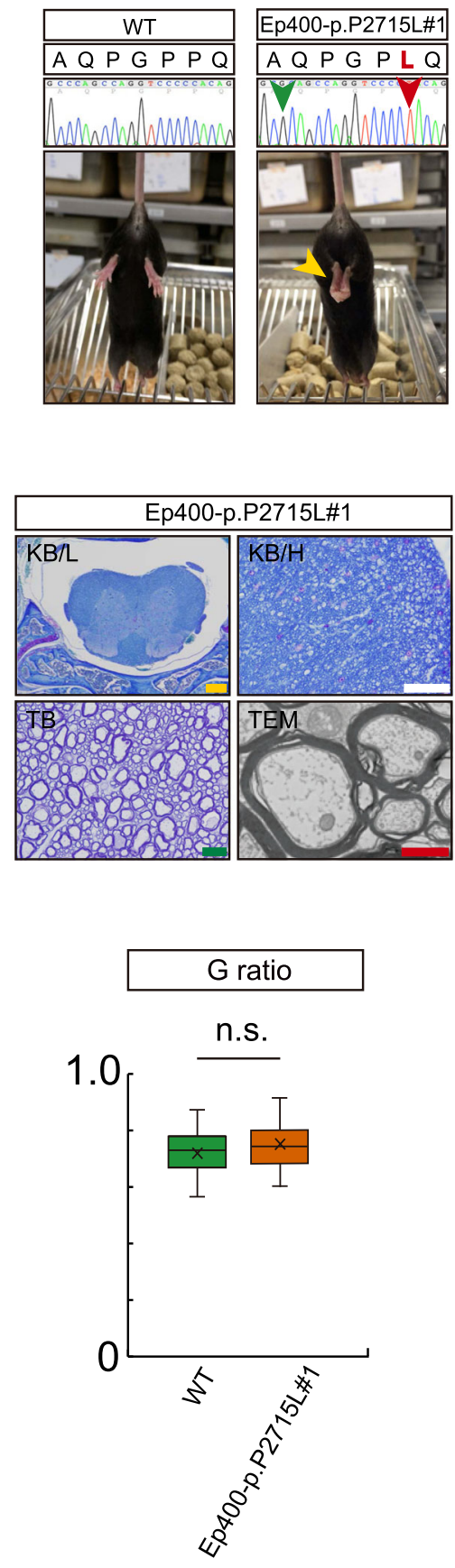

Fig. 2 Screening analysis of known phenotypes in Ep400-p.P2715L mice. a Body weight of Ep400-p.P2715L\#1 mice and their WT littermates (p. P2715L\#1 mice: $n=6$, WT: $n=6$ ). There was no significant difference in body weight in adult mice. $\mathbf{b}$ Genotype and hindlimb clasping. Sanger sequencing was used to confirm the missense mutation (p.P2715L, red arrow) and synonymous mutation to break the protospacer adjacent motif (PAM; green arrow) in Ep400. Hindlimb clasping (yellow arrow) was used as a marker of CNS dysfunction (p.P2715L\#1 mice: $n=6$, WT: $n=6$ ). c In a low-power field, there was no obvious differences in KB-stained tissue between 3-month-old Ep400-p.P2715L\#1 mice and their WT littermates. Ep400-p.P2715L\#1 mice showed a decrease in axon diameters in the spinal marrow by both KB and TB staining in a high-power field. Ep400-p. P2715L\#1 mice did not show any morphological abnormalities in myelin sheaths in the TEM analysis (p.P2715L\#1 mice: $n=2, W T: n=2$ ). KB/L= Klüver-Barrera staining, in a low-power field. $\mathrm{KB} / \mathrm{H}=$ Klüver-Barrera staining, in a high-power field. $\mathrm{TB}=$ Toluidine blue staining. Yellow scale bar $=$ $200 \mu \mathrm{m}$. White scale bar $=50 \mu \mathrm{m}$. Green scale bar $=10 \mu \mathrm{m}$. Red scale bar $=1 \mu \mathrm{m}$. $\mathbf{d}$ TEM analysis was used to compare axon diameters and G-ratios between Ep400-p.P2715L\#1 and WT mice. A significant decrease in axon diameter in Ep400-p.P2715L\#1 mice was confirmed by TEM analysis. Ep400p.P2715L\#1 mice did not show significant differences in G-ratios compared with their WT littermates (p.P2715L\#1 mice: $n=2$, WT: $n=2$ ). 
EP400 is expressed in all cell types in the developing rodent CNS, and EP400-dependent SWR chromatin remodelling activity is required for myelination. Hypomyelination and myelin abnormalities have been previously reported in the central and peripheral nervous systems of oligodendrocyte-specific Ep400 conditionalknockout mice ${ }^{52,53}$. Of note, we observed decreased axon diameters in the knock-in mice compared with the WT mice in $\mathrm{KB}$ and $\mathrm{TB}$ staining. However, there were no morphological abnormalities in the myelin sheaths of Ep400-p.P2715L\#1 mice (Fig. 2c). Axon diameters were significantly decreased in the Ep400-p.P2715L\#1 mice under TEM $\left(\mathrm{WT}_{\text {average }}=4.9313 \mu \mathrm{m}, \quad \mathrm{Ep} 400-\mathrm{p}\right.$. P2715L $\# 1_{\text {average }}=3.735 \mu \mathrm{m}$; Welch's $t$-test, $P=3.71769 \mathrm{E}$ 05; Fig. 2d). However, TEM analysis indicated no differences in G-ratios (diameter of naked axon/diameter of myelinated axon) in the Ep400-p.P2715L\#1 mice compared with their WT littermates $\left(\mathrm{WT}_{\text {average }}=0.7143\right.$, Ep400-p.P2715L\# $1_{\text {average }}=0.7506$; Student's $t$-test, $P=$ 0.0732; Fig. 2d). The histological features of Ep400-p. P2715L\#2 mice were the same as those of Ep400-p. P2715L\#1 mice (Supplementary Fig. 2b, c).

\section{HE staining of CNS tissue from Ep400-p.P2715L mice}

We used histological methods to examine morphological abnormalities in the CNS of Ep400-p.P2715L\#1 mice, and observed no clear differences (e.g., abnormalities in cortical structure, neuronal dropout, or cortical atrophy) in HE-stained tissue from these mice compared with their WT littermates (Supplementary Fig. 3a, b).

\section{Neurobehavioural screening battery in Ep400-p.P2715L mice}

To date, there have been no reports of the effects of Ep400 dysfunction on neurobehavioural phenotypes. Thus, to evaluate the potential impact of EP400-p.P2715L protein expression in the CNS on neurobehavioral phenotypes, we screened for neurobehavioural abnormalities using a neurobehavioural screening battery, which consisted of four different tests (the open-field, Y-maze, rotarod, and social interaction tests). The open-field test showed no significant differences in behaviour between 3month-old Ep400-p.P2715L\#1 mice and WT littermates. However, 6-month-old Ep400-p.P2715L\#1 mice showed significantly decreased central time $\left(\mathrm{WT}_{\text {average }}=36.8 \mathrm{~s}\right.$, Ep400-p.P2715L $\# 1_{\text {average }}=20.4 \mathrm{~s} ;$ Student's $t$-test, $P=$ 0.018 ; Fig. 3a). The social interaction test also revealed no significant differences between 3-month-old Ep400-p. P2715L\#1 mice and WT littermates. In contrast, 6month-old Ep400-p.P2715L\#1 mice hesitated to approach the stimulator mice and showed a significantly increased number of stretched approaches compared with WT littermates $\left(\mathrm{WT}_{\text {average }}=0.333\right.$, Ep400-p.P2715L $\# 1_{\text {average }}=$ 2.5; Student's $t$-test, $P=0.0265$; Fig. $3 \mathrm{~b}$, Supplementary
Video 2). These data suggest that Ep400-p.P2715L\#1 mice develop anxiety-like behaviours with age. The Ep400-p. P2715L\#1 mice showed no significant behavioural abnormalities in the Y-maze or rotarod tests at any age (Fig. 3c, d).

\section{Discussion}

We identified a possible novel schizophrenia-causing gene in a Japanese family containing multiple members affected with severe schizophrenia/TRS. Using WES, a unique missense variant in EP4OO was identified that completely segregated with disease in this family.

Few studies have reported an association between EP400 and psychiatric disorders. Prados et al. reported that a DNA methylation status change of EP400 was associated with borderline personality disorder ${ }^{54}$. However, no study has yet reported an association between EP400 and schizophrenia. We performed a case-control analysis to investigate the association between EP400 deleterious mutations and schizophrenia. The singlevariant association test indicated that deleterious variants in EP400 were not significantly associated with schizophrenia. Moreover, in gene-based rare-variant association tests, EP400 did not show significant enrichment of rare variants in schizophrenia. These results, together with the absence of the identified familial mutation (rs200626129) in 285 schizophrenia patients, suggest that rare variants in EP400 do not account for a large subgroup of schizophrenia patients.

Recent investigations have demonstrated the extreme difficulty of identifying individual genes with significant associations in rare-variant association studies ${ }^{17,18}$. For example, Genovese et al.$^{55}$ failed to implicate any specific gene (including EP400) in schizophrenia, and only one exome study to date, which investigated approximately 15,000 exomes, has been successful in reporting an individual risk gene (SETD1A) for schizophrenia ${ }^{15}$. Given these findings, the present study likely lacked the statistical power needed to support rare-variant accumulation because of its small sample size. Our study concept was based on the rare variants' contribution with relatively high impact effect; thus, a large sample size is necessary for rare-variant association studies ${ }^{56}$. To explore the association between rare EP400 variants and schizophrenia via rare-variant association studies, further analyses with larger sample sizes $(>10,000$, with multi-ethnic populations) are required.

Ep400-p.P2715L mice, corresponding to the human P2805L mutation, showed anxiety-like behaviour and had decreased axon diameters in the spinal cord white matter. These results reflect the successful use of mice to demonstrate that the mouse P2715L mutation causes histological and neurobehavioural abnormalities, which may help to explain the pathogenesis of the human 


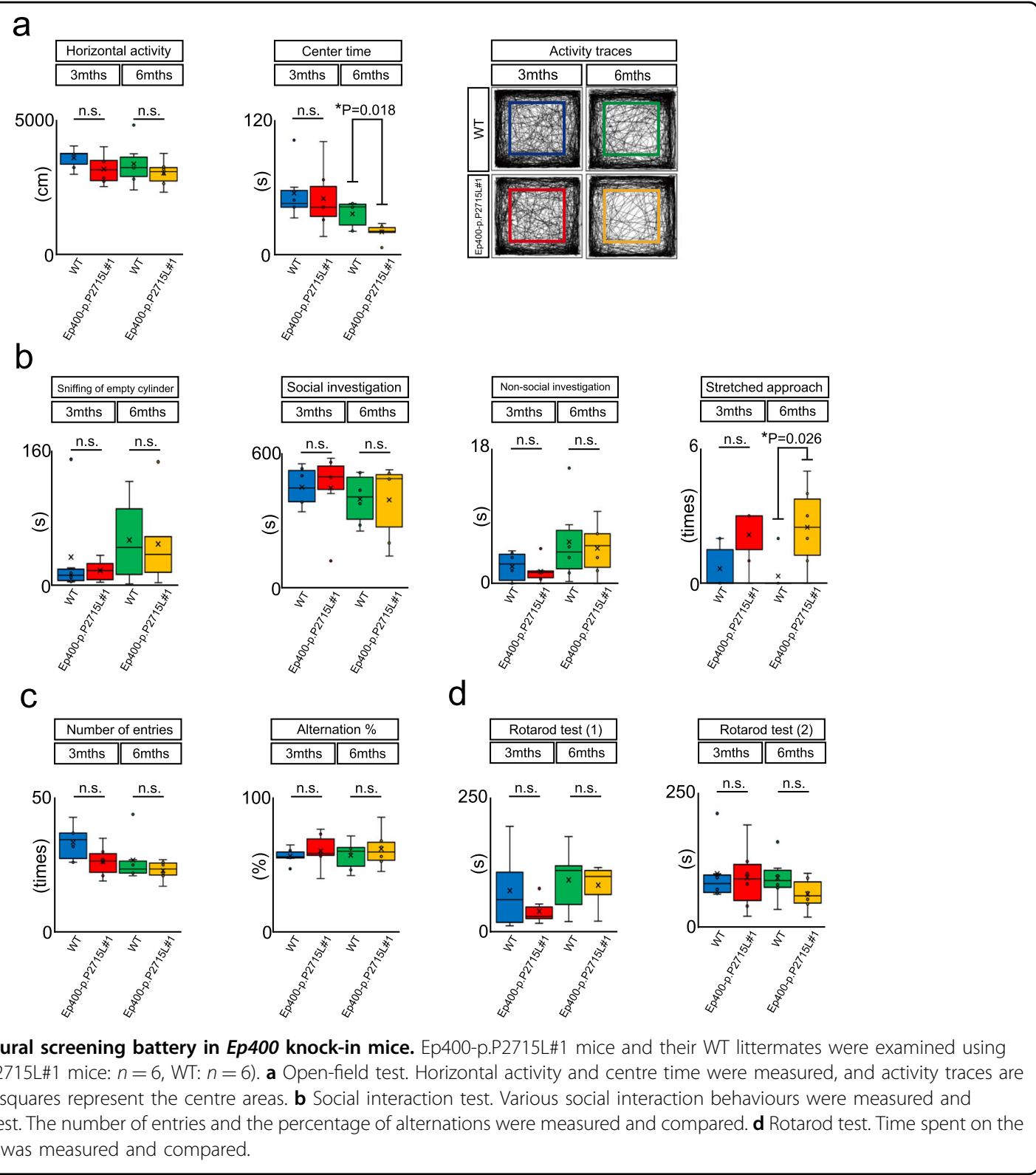

P2805L mutation in the family that was investigated in the present study. Anxiety is not a main symptom of schizophrenia but is sometimes observed in patients with this disease $^{57,58}$. In addition, some studies have reported myelin and axon abnormalities in the white matter of schizophrenia patients ${ }^{59,60}$. These reports, therefore, indicate that our mouse model may mimic at least some of the biological aetiologies of this family. However, the phenotypes observed in Ep400 gene-edited mice in the present study are not likely to be easily generalisable, especially considering that the burden of rare variants in EP400 on the development of schizophrenia remains unclear. In addition, because the present study was limited to screening only, it is necessary to analyse whether there are any unknown phenotypes in these mice, and whether they show appropriate phenotypes to be considered a model of schizophrenia.

EP400 is the central ATP-hydrolysing subunit of the TIP60/EP400 complex ${ }^{61,62}$, which is thought to exchange histone $\mathrm{H} 2 \mathrm{~A}$ for $\mathrm{H} 2 \mathrm{~A} . \mathrm{Z}$ in nucleosomes (especially in gene regulatory regions such as promoters ${ }^{63-65}$ ) to influence gene expression and DNA repair ${ }^{66}$. However, the molecular functions of EP400 in the CNS are poorly characterised. Elsesser et al. reported that EP400 interacts with the transcription factor SOX10, binds to myelin regulatory factor (MYRF) gene regulatory regions, and induces this central transcriptional regulator of myelination ${ }^{52}$. Mutual interactions between oligodendrocytes and neurons control the myelination and maturation of neural axons ${ }^{67-69}$. Accordingly, the p.P2715L mutation might decrease axon 
diameter via the dysfunction of these mutual interactions between oligodendrocytes and neurons in the developing CNS. In addition, the p.P2715L mice partially replicated the phenotype of Ep400 conditional-knockout mice that had been reported previously. This result, together with the finding that p.P2715L mice show a novel phenotypeof reduced axon diameter-suggests that the p.P2715L mutation may not simply cause a loss of function of the EP400 protein (e.g., a gain-of-function or partial-loss-offunction mutation). Further functional studies that confirm the abnormal functions of mutated EP400 in the developing CNS are, therefore, required to reveal the underlying pathogenic mechanisms.

In conclusion, we identified a unique missense variant in EP400 through the exome sequencing of a Japanese family containing multiple schizophrenia patients. The histological and neurobehavioural abnormalities observed in Ep400 gene-edited mice implicate the pathogenesis of this familial mutation in the schizophrenia multiplex family. The phenotypes of the Ep400 gene-edited mice may not be generalisable, because the burden of rare variants in EP400 on the development of schizophrenia remains unclear. However, a better understanding of the functional effects of EP400 in the CNS might help to clarify both the pathophysiology of schizophrenia and the development of new therapeutic targets for this disease.

\section{Acknowledgements}

We thank the patients and their family members for participating in this study. We also thank Prof. Yoshibumi Nakane, Prof. Hideyuki Nakane, Dr. Sumitaka Matumoto, Dr. Kazutaka Matumoto, Dr. Hironori Hayashida, Dr. Kosuke Fujimaru, Dr. Takefusa Yonashiro, Dr. Kazuo Sakamoto, Dr. Ikuro Hironaka, and Dr. Takahiro Tsujita for their cooperation in collecting samples, as well as Ms. Chisa Koga, Ms. Yasuko Noguchi, Ms. Yukie Takahashi, Mr. Hitomi Yano, Mr. Takahiro Motoyama, and Dr. Hitomi Shimizu for their technical support in the analysis. We also thank J. Ludovic Croxford, PhD, and Bronwen Gardner, PhD, from Edanz Group (https://en-author-services.edanz.com/ac) for editing a draft of this manuscript. This work was supported by JSPS KAKENHI (grant numbers JP26461721, JP16K10190, and JP19K08050), as well as by the Takeda Science Foundation (to Y.M.).

\footnotetext{
Author details

${ }^{1}$ Department of Neuropsychiatry, Unit of Translation Medicine, Nagasaki University Graduate School of Biomedical Sciences, Nagasaki, Japan. ${ }^{2}$ Child and Adolescent Psychiatry Community Partnership Unit, Nagasaki University Hospital, Nagasaki, Japan. ${ }^{3}$ Department of Human Genetics, Nagasaki University Graduate School of Biomedical Sciences, Nagasaki, Japan. ${ }^{4}$ Department of Infectious Diseases, Nagasaki University Hospital, Nagasaki, Japan. ${ }^{5}$ Department of Cell Pathology, Graduate School of Medical Sciences, Kumamoto University, Kumamoto, Japan. ${ }^{6}$ Department of Clinical Psychology, Faculty of Medicine, Kagawa University, Kagawa, Japan. ${ }^{7}$ Gene Research Center, Center for Frontier Life Sciences, Nagasaki University, Nagasaki, Japan.

${ }^{8}$ Michino-o Hospital, Nagasaki, Japan
}

\section{Author contributions}

Y.M., S.O., and K.Y. designed the experiments. Y.M., S.Y., A.K., H.M., A.I., T.T., Y.K., H.O., N. K., T.K., Y.O., S.O., and K.Y. performed the experiments and data analysis. Y.M. and S.O. wrote the paper. K.Y. and S.O. supervised the research.

\section{Data availability}

All relevant data supporting the findings of this study are available within the article and its Supplementary Information files, or from the corresponding authors upon reasonable request. Source data are provided with this paper.

\section{Conflict of interest}

The authors declare no competing interests.

\section{Ethics}

Human subjects: Experimental procedures were approved by the Committee for the Ethical Issues on Human Genome and Gene Analysis at Nagasaki University. The methods were carried out in accordance with the Ethical Guidelines for Medical and Health Research Involving Human Subjects. Informed consent was obtained from all participants in this study. Mouse subjects: Experimental procedures were approved by the Ethics Review Committee for Animal Experimentation at Nagasaki University.

\section{Publisher's note}

Springer Nature remains neutral with regard to jurisdictional claims in published maps and institutional affiliations.

Supplementary information The online version contains supplementary material available at https://doi.org/10.1038/s41398-021-01258-1.

Received: 24 December 2020 Revised: 10 January 2021 Accepted: 27 January 2021

Published online: 18 February 2021

\section{References}

1. Pisanu, C. \& Squassina, A. Treatment-resistant schizophrenia: insights from genetic studies and machine learning approaches. Front. Pharmacol. 10, 617 (2019).

2. Gouvêa-Junqueira, D. et al. Novel treatment strategies targeting myelin and oligodendrocyte dysfunction in schizophrenia. Front. Psychiatry 11, 379 (2020).

3. Fulford, D., Campellone, T. \& Gard, D. E. Social motivation in schizophrenia: how research on basic reward processes informs and limits our understanding. Clin. Psychol. Rev. 63, 12-24 (2018).

4. Cotter, J., Barnett, J. H. \& Granger, K. The use of cognitive screening in pharmacotherapy trials for cognitive impairment associated with schizophrenia. Front. Psychiatry 10, 648 (2019).

5. Laursen, T. M. Causes of premature mortality in schizophrenia: a review of literature published in 2018. Curr. Opin. Psychiatry 32, 388-393 (2019).

6. Kessler, R. C., Chiu, W. T., Demler, O., Merikangas, K. R. \& Walters, E. E. Prevalence, severity, and comorbidity of 12-month DSM-IV disorders in the National Comorbidity Survey Replication. Arch. Gen. Psychiatry 62, 617-627 (2005).

7. Vita, A. et al. Treatment-resistant schizophrenia: genetic and neuroimaging correlates. Front. Pharmacol. 10, 402 (2019).

8. Radaic, A. \& Martins-de-Souza, D. The state of the art of nanopsychiatry for schizophrenia diagnostics and treatment. Nanomedicine 28, 102222 (2020).

9. Sullivan, P. F., Kendler, K. S. \& Neale, M. C. Schizophrenia as a complex trait: evidence from a meta-analysis of twin studies. Arch. Gen. Psychiatry 60, 1187-1192 (2003)

10. Bryois, J. et al. Evaluation of chromatin accessibility in prefrontal cortex of individuals with schizophrenia. Nat. Commun. 9, 3121 (2018).

11. Golov, A. K., Kondratyev, N. V., Kostyuk, G. P. \& Golimbet, A. V. E. Novel approaches for identifying the molecular background of schizophrenia. Cells $\mathbf{9}$, 246 (2020).

12. Power, R. A. et al. Fecundity of patients with schizophrenia, autism, bipolar disorder, depression, anorexia nervosa, or substance abuse vs their unaffected siblings. JAMA Psychiatry 70, 22-30 (2013).

13. Bustamante, M. L. et al. Shifting the focus toward rare variants in schizophrenia to close the gap from genotype to phenotype. Am. J. Med. Genet. B Neuropsychiatr. Genet. 174, 663-670 (2017).

14. Steinberg, S. et al. Truncating mutations in RBM12 are associated with psychosis. Nat. Genet. 49, 1251-1254 (2017).

15. Singh, T. et al. Rare loss-of-function variants in SETD1A are associated with schizophrenia and developmental disorders. Nat. Neurosci. 19, 571-577 (2016). 
16. Rees, E. et al. De novo mutations identified by exome sequencing implicate rare missense variants in SLC6A1 in schizophrenia. Nat. Neurosci. 23, 179-184 (2020).

17. Coelewij, L. \& Curtis, D. Mini-review: update on the genetics of schizophrenia. Ann. Hum. Genet. 82, 239-243 (2018).

18. Gratten, J. Rare variants are common in schizophrenia. Nat. Neurosci. 19, 1426-1428 (2016)

19. Lehman, A. F. et al. Practice guideline for the treatment of patients with schizophrenia, second edition. Am. J. Psychiatry 161, 1-56 (2004).

20. McKenna, A. et al. The Genome Analysis Toolkit: a MapReduce framework for analyzing next-generation DNA sequencing data. Genome Res. 20, 1297-1303 (2010).

21. Van der Auwera, G. A. et al. From FastQ data to high confidence variant calls: the Genome Analysis Toolkit best practices pipeline. Curr. Protoc. Bioinforma. 43, 11.10.1-11.10.33 (2013).

22. Wang, K., Li, M. \& Hakonarson, H. ANNOVAR: functional annotation of genetic variants from high-throughput sequencing data. Nucleic Acids Res. 38, e164 (2010).

23. Karczewski, K. J. et al. The mutational constraint spectrum quantified from variation in 141,456 humans. Nature 581, 434-443 (2020).

24. Higasa, K. et al. Human genetic variation database, a reference database of genetic variations in the Japanese population. J. Hum. Genet. 61, 547-553 (2016).

25. Rozen, S. \& Skaletsky, H. Primer3 on the WWW for general users and for biologist programmers. Methods Mol. Biol. 132, 365-386 (2000).

26. Zhao, $\mathrm{H}$. et al. CrossMap: a versatile tool for coordinate conversion between genome assemblies. Bioinformatics 30, 1006-1007 (2014).

27. Kumar, P., Henikoff, S. \& Ng, P. C. Predicting the effects of coding nonsynonymous variants on protein function using the SIFT algorithm. Nat. Protoc. 4, 1073-1081 (2009)

28. Choi, Y., Sims, G. E., Murphy, S., Miller, J. R. \& Chan, A. P. Predicting the functional effect of amino acid substitutions and indels. PLOS ONE 7, e46688 (2012).

29. Schwarz, J. M., Cooper, D. N., Schuelke, M. \& Seelow, D. MutationTaster2: mutation prediction for the deep-sequencing age. Nat. Methods 11, 361-362 (2014).

30. Jagadeesh, K. A. et al. M-CAP eliminates a majority of variants of uncertain significance in clinical exomes at high sensitivity. Nat. Genet. 48, 1581-1586 (2016).

31. Dunker, A. K. et al. The unfoldomics decade: an update on intrinsically disordered proteins. BMC Genomics 9, S1 (2008).

32. Garner, E., Cannon, P., Romero, P., Obradovic, Z. \& Dunker, A. K. Predicting disordered regions from amino acid sequence: common themes despite differing structural characterization. Genome Inform. Ser. Workshop Genome Inform. 9, 201-213 (1998).

33. Garner, E., Romero, P., Dunker, A. K., Brown, C. \& Obradovic, Z. Predicting binding regions within disordered proteins. Genome Inform. Ser. Workshop Genome Inform. 10, 41-50 (1999).

34. Li, B. \& Leal, S. M. Methods for detecting associations with rare variants for common diseases: application to analysis of sequence data. Am. J. Hum. Genet. 83, 311-321 (2008).

35. Madsen, B. E. \& Browning, S. R. A groupwise association test for rare mutations using a weighted sum statistic. PLoS Genet. 5, e1000384 (2009).

36. Lee, S. et al. Optimal unified approach for rare-variant association testing with application to small-sample case-control whole-exome sequencing studies. Am. J. Hum. Genet. 91, 224-237 (2012).

37. 1000 Genomes Projects Consortium. et al. A global reference for human genetic variation. Nature 526, 68-74 (2015).

38. Lek, M. et al. Analysis of protein-cording genetic variation in 60,706 humans. Nature 536, 285-291 (2016).

39. Higasa, K. et al. Human genetic variation database, a reference database of genetic variations in Japanese population. J. Hum. Genet. 61, 547-553 (2016).

40. Schneider, C. A., Rasband, W. S. \& Eliceiri, K. W. NIH Image to ImageJ: 25 years of image analysis. Nat. Methods 9, 671-675 (2012)

41. Rueden, C. T. et al. ImageJ2: Image for the next generation of scientific image data. BMC Bioinforma. 18, 529 (2017).

42. Thomas, P. S. Jr et al. Loss of endogenous androgen receptor protein accelerates motor neuron degeneration and accentuates androgen insensitivity in a mouse model of X-linked spinal and bulbar muscular atrophy. Hum. Mol. Genet. 15, 2225-2238 (2006)

43. Lalonde, R. \& Strazielle, C. Brain regions and genes affecting limb-clasping responses. Brain. Res. Rev. 67, 252-259 (2011).

44. Guyenet, S. J. et al. A simple composite phenotype scoring system for evaluating mouse models of cerebellar ataxia. J. Vis. Exp. 39, 1787 (2010).

45. Kraeuter, A. K., Guest, P. C. \& Sarnyai, Z. The open field test for measuring locomotor activity and anxiety-like behavior. Methods Mol. Biol. 1916, 99-103 (2019).

46. Weinstock, M. Prenatal stressors in rodents: effects on behavior. Neurobiol Stress 6, 3-13 (2016).

47. Friard, O. \& Gamba, M. BORIS: a free, versatile open-source event-logging software for video/audio coding and live observations. Methods Ecol. Evol. 7 1325-1330 (2016)

48. Adams, W., Kusljic, S. \& van den Buuse, M. Serotonin depletion in the dorsal and ventral hippocampus: effects on locomotor hyperactivity, prepulse inhibition and learning and memory. Neuropharmacology 55, 1048-1055 (2008).

49. Deacon, R. M. Measuring motor coordination in mice. J. Vis. Exp. 75, e2609 (2013).

50. Hawrylycz, M. J. et al. An anatomically comprehensive atlas of the adult human brain transcriptome. Nature 489, 391-399 (2012).

51. Lein, E. S. et al. Genome-wide atlas of gene expression in the adult mouse brain. Nature 445, 168-176 (2007).

52. Elsesser, O. et al. Chromatin remodeler Ep400 ensures oligodendrocyte survival and is required for myelination in the vertebrate central nervous system. Nucleic Acids Res. 47, 6208-6224 (2019).

53. Fröb, F. et al. Ep400 deficiency in Schwann cells causes persistent expression of early developmental regulators and peripheral neuropathy. Nat. Commun. 10, 2361 (2019)

54. Prados, J. et al. Borderline personality disorder and childhood maltreatment: a genome-wide methylation analysis. Genes Brain Behav. 14, 177-188 (2015).

55. Genovese, G. et al. Increased burden of ultra-rare protein-altering variants among 4,877 individuals with schizophrenia. Nat. Neurosci. 19, 1433-1441 (2016).

56. Moutsianas, L. et al. The power of gene-based rare variant methods to detect disease-associated variation and test hypotheses about complex disease. PLoS Genet. 11, e1005165 (2015).

57. Achim, A. M. et al. How prevalent are anxiety disorders in schizophrenia? A meta-analysis and critical review on a significant association. Schizophr. Bull. 37 811-821 (2011).

58. Lysaker, P. H. \& Salyers, M. P. Anxiety symptoms in schizophrenia spectrum disorders: associations with social function, positive and negative symptoms, hope and trauma history. Acta Psychiatr. Scand. 116, 290-298 (2007).

59. Du, F. et al. Myelin and axon abnormalities in schizophrenia measured with magnetic resonance imaging techniques. Biol. Psychiatry 74, 451-457 (2013).

60. Tkachev, D. et al. Oligodendrocyte dysfunction in schizophrenia and bipolar disorder. Lancet 362, 798-805 (2003).

61. Hota, S. K. \& Bruneau, B. G. ATP-dependent chromatin remodeling during mammalian development. Development 143, 2882-2897 (2016).

62. Venkatesh, S. \& Workman, J. L. Histone exchange, chromatin structure and the regulation of transcription. Nat. Rev. Mol. Cell Biol. 16, 178-189 (2015).

63. Kusch, T. et al. Acetylation by Tip60 is required for selective histone variant exchange at DNA lesions. Science 306, 2084-2087 (2004).

64. Mizuguchi, G. et al. ATP-driven exchange of histone H2AZ variant catalyzed by SWR1 chromatin remodeling complex. Science 303, 343-348 (2004).

65. Pradhan, S. K. et al. EP400 deposits H3.3 into promoters and enhancers during gene activation. Mol. Cell 61, 27-38 (2016).

66. Lu, P. Y., Lévesque, N. \& Kobor, M. S. NuA4 and SWR1-C: two chromatinmodifying complexes with overlapping functions and components. Biochem. Cell Biol. 87, 799-815 (2009).

67. Shimizu, T., Osanai, Y. \& Ikenaka, K. Oligodendrocyte-neuron interactions: impact on myelination and brain function. Neurochem. Res. 43, 190-194 (2018).

68. Lee, S. et al. A culture system to study oligodendrocyte myelination processes using engineered nanofibers. Nat. Methods 9, 917-922 (2012).

69. Colello, R. J., Pott, U. \& Schwab, M. E. The role of oligodendrocytes and myelin on axon maturation in the developing rat retinofugal pathway. J. Neurosci. 14, 2594-2605 (1994) 